\title{
Single-path versus Multi-path Advance Reservation in Media Production Networks
}

\author{
Maryam Barshan, Hendrik Moens, Bruno Volckaert, Filip De Turck \\ Department of Information Technology, Ghent University - iMinds \\ Gaston Crommenlaan 8/201, B-9050 Gent, Belgium \\ Email: maryam.barshandintec.ugent.be
}

\begin{abstract}
In media production, a set of actors works simultaneously on video content from different sources. If the actors are geographically spread, the use of a shared substrate network can improve their collaborative efficiency. In such a network traffic consists mostly of large video files, which need to be transferred respecting strict deadlines. Restrictions on the underlying network can force the use of single-path routing mechanisms over multi-path approaches. In this paper, we investigate the influence of using single-path routing compared to multi-path routing in deadline-aware advance reservation (AR) systems for media production networks. We have modified our previously designed optimal multi-path advance reservation model to incorporate the single-path mechanism and heuristic alternatives are presented and thoroughly evaluated. The experimental results show that the single-path optimal model can only provide satisfactory performance when the network is not in contention. With the heuristic approach, when adequate bandwidth is provided, the multi-path approach outperforms the single-path by up to $7.3 \%$.

Index Terms-Advance bandwidth reservation, media production network, single-path, multi-path.
\end{abstract}

\section{INTRODUCTION}

Exchanging a large number of media files is daily business in media production companies of all types, ranging from production houses to broadcasters. Traditional ways of transporting data, i.e. using dedicated and expensive point to point high speed links or even using physical transportation systems (vehicles and accompanying human resources) is costly and highly inefficient. As a standard media transport medium, wide area IP-based shared networks are being used more and more within these media-centric process flows.

The work in this paper has been performed within the context of ICON MECaNO project, which aims to provide solutions for the transmission of large file-based media files and streaming sessions over IP-based network infrastructure, tailored to the quality and timing requirements of current and future media process flows.

In our previous work [1] we have shown the viability of using advance bandwidth reservation techniques in media production industry. We proposed an Integer Linear Programming (ILP) model to solve the AR scheduling problem. Based on this model, two static and dynamic scheduling algorithms were presented: the Static Advance Reservation Algorithm (SARA) which assumes all requests and their requirements are known in advance and the Dynamic Advance Reservation Algorithm (DARA) which supports rescheduling to incorporate new requests. In both algorithms we assumed that the flows can be split over multiple paths, making it possible to fully use the network. Using multi-path is however not always feasible when there is no full control over the underlying network and network devices. According to network flow theory, flows can be split into a number chunks, to be transferred over different paths through the network, this effect is undesired or even forbidden in some applications [2]. In the Internet, wireless networks, or overlay networks built on top of the Internet, traffic is mostly sent over a single path and generally splitting the flows is avoided due to the problem of packet reassembly at the receiver [3]. In addition, it is often not possible to use multi-path solutions due to limitations in the configuration of intermediate devices (e.g. the forwarding behavior on the routers cannot be modified to support multi-path routing).

While multi-path approaches are not always feasible, single path reservation cannot always meet the end-to-end QoS requirements in bandwidth-limited networks [4]. In this paper, we analyse the impact and importance of supporting multipath network flows in media production networks. To achieve this we have provided a modified version of the previously designed ILP-based models and algorithms to support unsplittable flows. This allows us to compare the performance of our advance network reservation system, using single-path versus multi-path bandwidth reservation approaches, to determine the importance of supporting multi-path flows.

The remainder of this paper is structured as follows. In Section II, we discuss related work. Section III, provides extensions to our models to allow for single path reservations. The heuristic-based AR scheduling algorithms are described in Section IV. Section V provides simulation results, comparing the proposed algorithms. Finally, Section VI concludes the paper.

\section{RELATED WORK}

The AR scheduling problem has been well studied in literature. While some have focused on rescheduling [5], [6], [7] and multi domain reservation [8], others have paid particular attention to real-world deployments [9], [10], [11], [12], and WDM optical networks [13]. However, according to Charbonneau et al. [13], only two advance reservation algorithms support elastic reservation, and both consider fixed 
start time for the flows, while we consider flexible flow start times.

Moreover, the problem addressed in this work is related to the multi-commodity flow problem (MCFP). Comprehensive surveys on the approaches to solve multi-commodity flow problems (MCFP) and their variants are provided in [14], [15]. In [16], unsplittable flow and single path MCFPs are studied. Our approach extends this by dealing with the problem of flow variation over time and solves an MCFP as a subproblem. Dynamic flows or flow variation over time are primarily introduced by Ford and Fulkerson [17], [18]. Our work differs by introducing support for variable reservations over time, elastic reservations, flexible start times and dependencies among different flows.

Another work [19] has compared single-path and multi-path routing approaches and concluded that multi-path routing provided limited gain compared to single-path routing. However, there are multiple differences which make their comparison inapplicable to the problem addressed in this paper. First, their investigation is not about advance reservation, but about routing under certain traffic conditions. The authors focus on a comparison when all node-pairs generate traffic, while we focus on large file transfers within large networks where only limited numbers of nodes act as source and sink nodes, and finally no transfer deadlines are present in their approach.

This work is an extension of our previous work [1], in which the multi-path version of ILP-based models with two objective functions are proposed and thoroughly evaluated. The MaxA objective function maximizes the request admittance ratio while the ASAP objective in addition to maximizing the number of admitted requests, also tries to schedule the requests as soon as possible. As results showed that ASAP outperforms MaxA by up to $3.27 \%$, in this paper evaluation is only performed for the ASAP objective function. In addition, the heuristic approaches are proposed and their performance is compared to the optimal algorithms.

\section{AR SCHEDULING MODEL}

In [1] we proposed a formal model for the advance reservation scheduling of network bandwidth. In this section we show how this model can be extended to perform single-path reservations.

The model can be used to schedule collections of requests, that consist of multiple interdependent and deadlineconstrained network transfers. Requests are grouped into scenarios, that represent a complex workflow. The workflows must be executed in their entirety, so when a scenario is admitted, all requests must be scheduled. The model only admits those scenarios for which sufficient bandwidth can be guaranteed during the reservation period. When a scenario is rejected, none of its requests are executed. The various requests within a scenario may depend on each other, meaning that one request can only start when other requests have finished. The model supports two types of network transfers: video streaming and large file transfers. The requests of all scenarios are stored in $R$. Consequently $R$ consists of both types. To make distinction between the two types $R_{f}$ which refers to file-based flows and $R_{s}$ which refers to the streaming requests are defined. The network is represented as a graph with nodes $N$ and edges $E$.

In this model the $n^{\text {th }}$ request is denoted by $r^{n}=$ $\left(s^{n}, d^{n}, t_{s}^{n}, t_{e}^{n}, i^{n}, b^{n}\right)$ comprising of the source of the request $s^{n}$, the destination node $d^{n}$, the time when the data for filebased request is ready to transfer $t_{s}^{n}$ (or fixed start time for video streaming request), the deadline for the transmission of the data of file-based request $t_{e}^{n}$ (or fixed end time for video streaming request), the duration of each request $i^{n}$ and finally the bandwidth demand of the request $b^{n}$. In particular, $r_{f}^{n}$ and $r_{s}^{n}$ refer to file-based and video streaming requests respectively. Moreover the volume of the files are denoted by $v^{n}$ and the time slot size by $I$. Table I lists other notations which have been used to define this extension.

TABLE I: Symbols and notations used in the formal model.

\begin{tabular}{ll}
\hline Variable & Description \\
\hline$\beta^{n, e, k}$ & Dedicated Bandwidth between link $e$, request $r^{n}$ and time slot $k$. \\
$A^{n}$ & Binary variable, 1 iff request $r^{n}$ is admitted, 0 otherwise. \\
$t_{s}^{\text {min }}$ & Minimum start time of all reservations. \\
$t_{e}^{\text {max }}$ & Maximum end time of all reservations. \\
$B^{e}$ & Bandwidth capacity of link $e$. \\
$E_{v}^{\text {out }}$ & This collection contains all edges starting from node $v$ (egress). \\
$E_{v}^{\text {in }}$ & This collection contains all edges ending in node $v$ (ingress). \\
\hline
\end{tabular}

This model is partially similar to the multi-path reservation model. All the decision variables, objective functions and constraints of former model are valid and applicable to this model. Therefore, only additions are discussed.

1) Additional decision variable: We need to make sure that only a single path is reserved for each request. To achieve this, in addition to the 6 decision variables, one more binary decision variable, $P^{n, e, k}$, is defined which indicates whether there is any reservation for request $n$ in time slot $k$ over link $e$.

$$
P^{n, e, k} \in[0,1] \quad \forall r^{n} \in R, \forall e \in E, k \in\left[t_{s}^{\min }, t_{e}^{\max }\right]
$$

2) Additional constraints: In addition to the constraints which ensure that capacity limitation, network flow concepts and dependencies among requests are respected, 5 extra constraints concerning single-path flow conservation of $P$ values and linking $P$ values to $\beta$ values are defined. Constraints 1,2 and 3 ensure that only a single-path is chosen to be reserved for a request during each time interval.

$$
\begin{aligned}
& \sum_{e \in E_{v}^{\text {out }}} P^{n, e, k}=\sum_{e \in E_{v}^{\text {in }}} P^{n, e, k} \\
& \forall r^{n} \in R, \forall k \in\left[t_{s}^{n}, t_{e}^{n}\right],\left\{\forall v \in N \mid v \notin\left\{s^{n}, d^{n}\right\}\right\} \\
& \sum_{e \in E_{s^{n}}^{\text {out }}} P^{n, e, k}=A^{n} \quad \forall r^{n} \in R, \forall k \in\left[t_{s}^{n}, t_{e}^{n}\right] \\
& \sum_{e \in E_{d^{n}}^{\text {in }}} P^{n, e, k}=A^{n} \quad \forall r^{n} \in R, \forall k \in\left[t_{s}^{n}, t_{e}^{n}\right]
\end{aligned}
$$



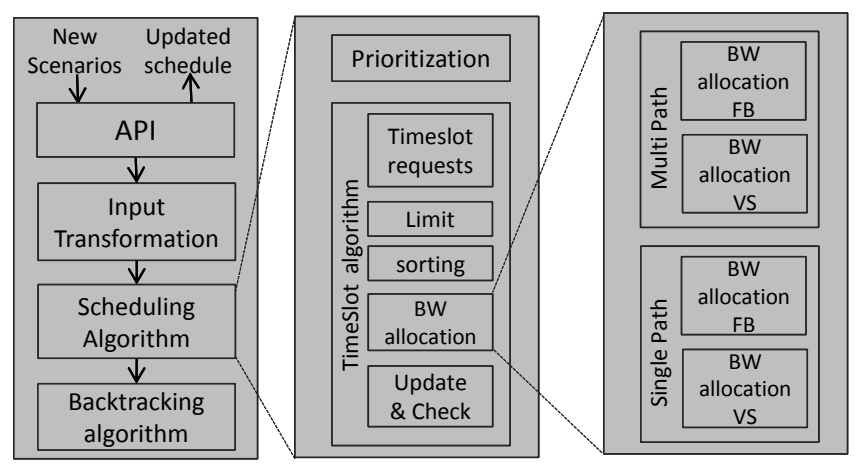

Fig. 1: Components of the Sequential Priority Based Advance Reservation Algorithm (SPB).

Constraint 4 ensures if the type of the request is video streaming, the dedicated and requested bandwidth must be equal. Also Constraint 5 is defined for linking $P$ values to $\beta$ flows.

$$
\begin{array}{r}
\beta^{n, e, k}=b^{n} \times P^{n, e, k} \forall e \in E, \forall r_{s}^{n} \in R_{s}, \forall k \in\left[t_{s}^{n}, t_{e}^{n}\right] \\
\beta^{n, e, k} \leq B^{e} \times P^{n, e, k} \forall e \in E, \forall r^{n} \in R, \forall k \in\left[t_{s}^{\text {min }}, t_{e}^{\text {max }}\right]
\end{array}
$$

\section{AR SCHEDULING ALGORITHM}

The Sequential Priority Based (SPB) advance reservation algorithm is a heuristic solution which is proposed due to the high computational overhead and scalability issue of the ILP approach. Individual components of the SPB algorithm are shown in Figure 1. As can be seen, new scenarios enter the reservation system through an API. In the next step any transformation can be applied. For example in the dynamic approach before the scheduling algorithm invocation, the previously admitted scenarios' demand needs to be updated. Then the scheduling algorithm is sequentially invoked for each scenario. If this process is successfully terminated the new scenario is admitted, and the schedule is updated. Otherwise, the previous schedule and network state remain untouched and the scenario is rejected. The scheduling algorithm consists of two components: The prioritization and the TimeSlot algorithms. The prioritization algorithm assigns priorities to the scenario's requests based on the estimated hard deadline and the volume. Since the deadline may not be specified for all requests, the latest possible deadline for those with no specific deadline should be estimated. Then all the scenario's requests are given to the TimeSlot algorithm. This algorithm consists of 5 sub-algorithms for each time interval.

TimeSlotRequests: First, the algorithm determines which requests can be served in the current time slot. For independent requests the algorithm looks at the start time. If the current interval is greater than or equal to the request start time, these requests are eligible to be added to the list of current requests. For requests with start time dependencies, the algorithm checks whether the requests on which this request depends are finished or not.

Limit: The limit for video streams is their required demand, which is fixed and non-variable and for file-based requests is their residual demand.

Sorting: In this step requests are sorted based on their previously assigned priorities.

BWallocation: We have defined four variations of bandwidth allocation algorithm for video streams and video files using single-path and multi-path routing. This algorithm first assigns cost to the network links using the Cost allocation component which tries to find the most desired paths and give them the highest cost. Then according to the approach and type of the request multi-path BWallocationFB, multi-path BWallocationVS, single-path BWallocationFB or single-path BWallocationVS algorithms is invoked.

Single-path BWallocation algorithms: The single-path $B$ Wallocation $F B$ algorithm is in charge of the FB requests. As we aim at transferring the video files in earlier timeslots, the single-path BWallocationFB algorithm first tries to find the most available bandwidth between the source and destination of the request. If multiple candidates with maximum bandwidth are available, this algorithm looks for the leastcost single-path that could carry this maximum flow using the modified version of Dijkstra to consider the cost of the paths, which are previously assigned to the network links, and ignoring the paths with lower capacity than the maximum flow.

The single-path BWallocationVS algorithm deals with video stream requests. For video streams, this algorithm first removes all the network links with capacities lower than the request demands, and then the least-cost path is determined. If no path is found, rescheduling is unsuccessful and the new scenario is rejected.

Multi-path BWallocation algorithms: The multi-path $B$ Wallocation $F B$ algorithm is based on maxflow and least-cost path algorithms. If the maxflow, which is calculated based on the Edmonds-Karp algorithm, is lower than the request limit, all the maxflow paths are reserved for this request. Otherwise, the algorithm forms a graph out of the maxflow paths and the k-shortest path is the second alternative. Finding the least-cost path is repeated until the total bandwidth offered by the paths is sufficient for the request.

The multi-path BWallocationVS algorithm iteratively looks for the least cost path on the whole graph and sums up the minimum available bandwidth of the paths. These steps are repeated over the residual graph while the total bandwidth provided by the paths fulfils the request demand.

Update and check feasibility: based on the provided result of the BWallocation component, and by calculating the residual demands, the requests requirements are updated and the feasibility of the results is checked. If the hard deadline of a request is reached, but part of the request has not been transferred yet and the residual demand is not zero, the hard deadline has not been met and rescheduling is infeasible. 


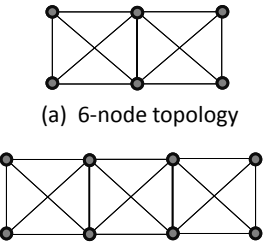

(b) 8-node topology

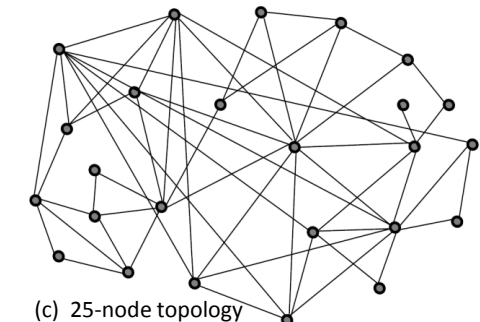

(c) 25-node topology

Fig. 2: Media production network topologies used in the evaluation.

\section{EXPERIMENTAL RESULTS}

This section evaluates the single-path and multi-path approaches for ILP-based and SPB scheduling algorithms. The influence of bandwidth availability, network load, and the time granularity are assessed.

\section{A. Evaluation Setup}

We found that the 12-node topology which is used in our previous evaluation yields identical results for the singlepath and multi-path approaches both for ILP-based and SPB algorithms. In this evaluation we have used 3 other topologies for media production networks which are depicted in Figure 2.

Throughout this section, $X X[Y Y, Z Z \%]$ denotes that approach $X X$ (i.e. ILP or SPB), routing mechanism $Y Y$ which can either be Single-Path (SP) or Multi-Path (MP) is used and ZZ\% of the use case instances are known at the start of the simulation. Each simulation run covers a 24 hour period. All results are averaged over 50 runs with different randomized inputs, error bars denote the standard error.

\section{B. ILP evaluation of single-path versus multi-path}

In this evaluation the number of use case instances equals 6 , resulting in of 62 requests. The 8-node topology and a fixed time interval granularity of 1 hour is used.

Figure 3 compares the percentage of admitted requests of ILP-based single-path and ILP-based multi-path approaches. From the figure, we can conclude that the multi-path approach significantly outperforms the single-path approach when network contention happens (bandwidth capacity lower than 200 Mbps). In this situation there is insufficient capacity for the scheduler to reserve a single-path for some flows by their deadline. However, this has no impact on the multi-path approach as the flows can be split and sent over multiple paths. The result shows that there is up to $24.3 \%$ differences in request admittance ratio.

\section{Comparison of ILP-based model with SPB approach}

For this evaluation the impact of network capacity is assessed. The 6-node network topology is used and the number of scenarios is 8 ( 85 requests in total). Figure 4 compares the ILP-based algorithms to the SPB ones for both multi-path and single-path approaches. This figure shows that the result of the SPB algorithm is within $13.6 \%$ of the ILP approach when

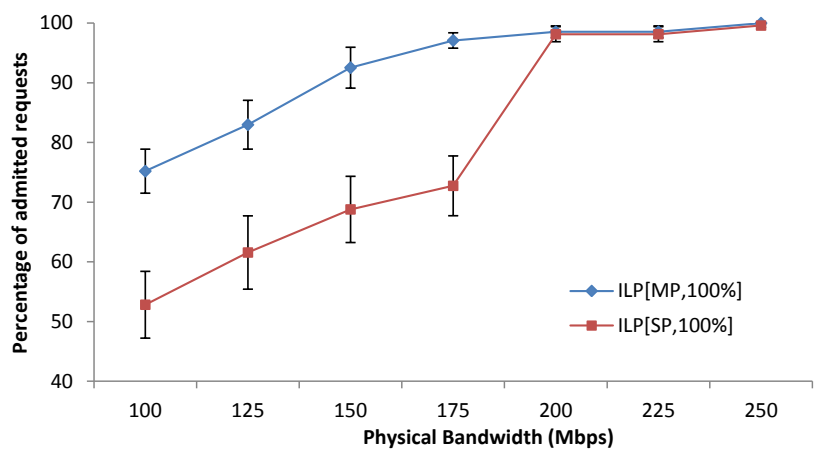

Fig. 3: Comparing single-path versus multi-path in ILP-based approach.

multi-path routing mechanisms are used. For the single-path method, a similar trend can be observed.

\section{Evaluation of single-path and multi-path in SPB approach}

In this section we evaluate the impact of network capacity, time slot granularity and network load on the performance of the SPB algorithms. Two topologies of the 8-node and 25node serve as media production infrastructures in Figure 5 and Figure 6 respectively.

In part (a) of both figures, the media network infrastructures have been configured for different available bandwidths to investigate the impact of network capacities on the performance of our algorithms. In both plots a time slot size of 1 hour is used and the number of scenarios is 20 (209 requests) and 50 (519 requests) for the 8-node and 25-node topologies respectively. The result shows that the performance of singlepath approach is within $7.3 \%$ and $6.7 \%$ of multi-path approach for 8-node and 25-node topologies respectively.

In part (b) the impact of time slot granularity is studied. While the number of use case instances is considered 20 and 50 for smaller and larger topologies respectively, a link capacity of $200 \mathrm{Mbps}$ is used in both evaluations. The results show that the experiment with shortest time slot leads to the best performance and the multi-path approach outperforms the

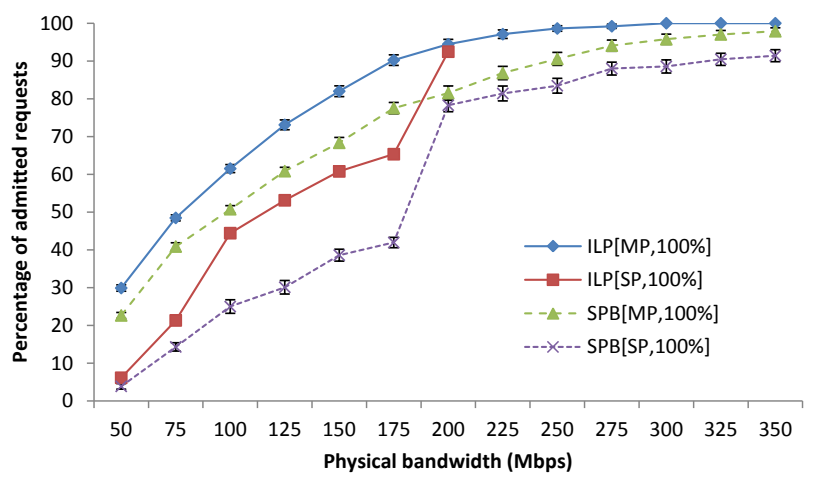

Fig. 4: Comparison of optimal ILP with SPB approach. 

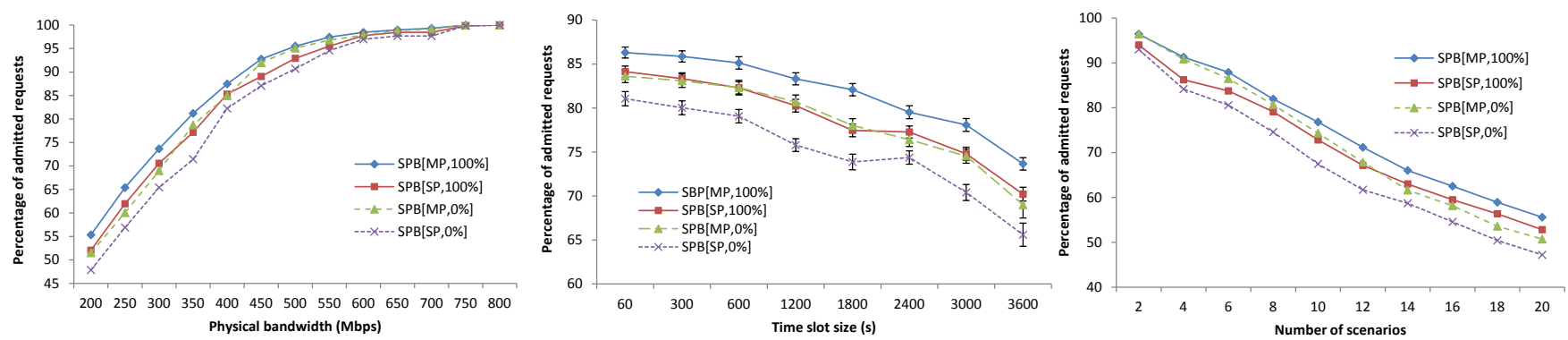

(a) Time slot size $=1$ hour, Number of scenarios=20(b) Bandwidth=200 Mbps, Number of scenarios=20

(c) Time slot size $=1$ hour, Bandwidth=300 Mbps

Fig. 5: Impact of bandwidth capacity,time slot granularity and network load on admission rate in 8-node topology

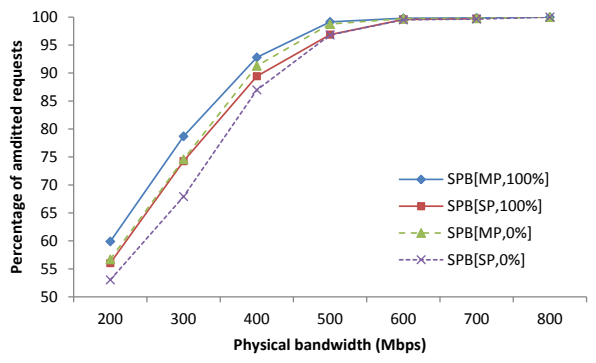

(a) Time slot size $=1$ hour, Number of scenarios $=50$

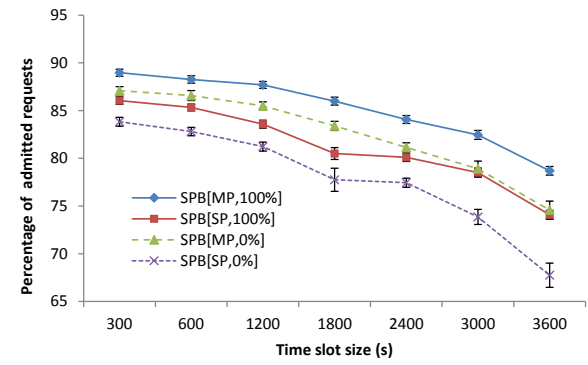

(b) Bandwidth $=200 \mathrm{Mbps}$, Number of scenarios $=50$

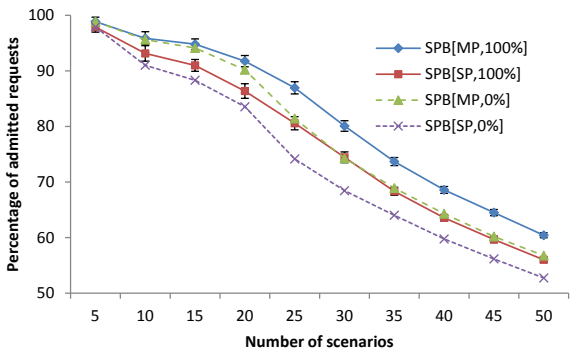

(c) Time slot size=1 hour, Bandwidth=300 Mbps

Fig. 6: Impact of bandwidth capacity, time slot granularity and network load on admission rate in a 25-node topology.

single-path approach up to $4.9 \%$ and $6.8 \%$ in Figure 5 and Figure 6 respectively.

Finally, part (c) evaluates the impact of network load when the number of scenarios increases up to 20 and 50 scenarios in Figure 5 and Figure 6 respectively. In both figures the timeslot size of 1 hour and network capacity of $300 \mathrm{Mbps}$ is used. The results show that for both smaller and larger topologies the multi-path approach outperforms the single-path approach by up to $6.9 \%$ and $7.3 \%$ respectively.

\section{CONCLUSION}

In this paper, the impact of using single-path routing mechanisms in advance reservation system for media production networks, is compared to a multi-path approach. We extended the optimal advance bandwidth reservation model to incorporate single-path routing and provided equivalent heuristic solutions. The impact of physical capacity, time interval granularity and network load were evaluated. Based on our simulation results, a multi-path approach is beneficial, improving the request admittance rate by up to $24.3 \%$ compared to when by using single-path solutions. However, if multi-path routing is not a viable solution, our evaluation showed that increasing the network capacity will significantly improve the performance of a single-path advance reservation system. The evaluation of our heuristics indicated that the single-path approach can achieve performance levels which remain within $7.3 \%$ of multipath routing mechanism.

\section{ACKNOWLEDGMENT}

The research leading to these results was performed within the context of ICON MECaNO. It is a project co-funded by iMinds, a digital research institute founded by the Flemish Government. Project partners are SDNsquare, Limecraft, VideoHouse, Alcatel-Lucent, and VRT, with project support from IWT under grant agreement no. 130646.

\section{REFERENCES}

[1] M. Barshan, H. Moens, J. Famaey, and F. De Turck, "Algorithms for advance bandwidth reservation in media production networks,"

[2] M. Martens and M. Skutella, "Flows on few paths: Algorithms and lower bounds," Networks, vol. 48, no. 2, pp. 68-76, 2006.

[3] B. Awerbuch, Y. Azar, and A. Epstein, "The price of routing unsplittable flow," in Proceedings of the thirty-seventh annual ACM symposium on Theory of computing, pp. 57-66, ACM, 2005.

[4] J. Chen, S.-H. Chan, and V. O. Li, "Multipath routing for video delivery over bandwidth-limited networks," Selected Areas in Communications, IEEE Journal on, vol. 22, no. 10, pp. 1920-1932, 2004.

[5] K. Rajah, S. Ranka, and Y. Xia, "Advance reservations and scheduling for bulk transfers in research networks," IEEE Trans. Parallel Distrib. Syst., vol. 20, pp. 1682-1697, Nov. 2009.

[6] C. Xie, H. Alazemi, and N. Ghani, "Rerouting in advance reservation networks," Computer Communications, vol. 35, no. 12, pp. 1411-1421, 2012.

[7] L. Zuo, M. M. Zhu, and C. Q. Wu, "Fast and efficient bandwidth reservation algorithms for dynamic network provisioning," Journal of Network and Systems Management, 2013.

[8] H. Alazemi, F. Xu, C. Xie, and N. Ghani, "Advance reservation in distributed multi-domain networks," IEEE Systems Journal, 2013.

[9] C. Guok, E. N. Engineer, and D. Robertson, "Esnet on-demand secure circuits and advance reservation system (oscars)," Internet2 Joint, 2006. 
[10] B. Gibbard, D. Katramatos, and D. Yu, "Terapaths: end-to-end network path qos configuration using cross-domain reservation negotiation," in Broadband Communications, Networks and Systems, 2006. BROAD NETS 2006. 3rd International Conference on, pp. 1-9, IEEE, 2006.

[11] J. Gu, D. Katramatos, X. Liu, V. Natarajan, A. Shoshani, A. Sim, D. Yu, S. Bradley, and S. McKee, "Stornet: Integrated dynamic storage and network resource provisioning and management for automated data transfers," in Journal of Physics: Conference Series, vol. 331, p. 012002, IOP Publishing, 2011.

[12] S. Sharma, D. Katramatos, D. Yu, and L. Shi, "Design and implementation of an intelligent end-to-end network qos system," in Proceedings of the International Conference on High Performance Computing, Networking, Storage and Analysis, SC '12, (Los Alamitos, CA, USA), pp. 68:1-68:11, IEEE Computer Society Press, 2012.

[13] N. Charbonneau and V. M. Vokkarane, "A survey of advance reservation routing and wavelength assignment in wavelength-routed wdm networks," Communications Surveys \& Tutorials, IEEE, vol. 14, no. 4, pp. 1037-1064, 2012.

[14] A. Ouorou, P. Mahey, and J.-P. Vial, "A survey of algorithms for convex multicommodity flow problems," Management science, vol. 46, no. 1, pp. 126-147, 2000.

[15] J. L. Kennington, "A survey of linear cost multicommodity network flows," Operations Research, vol. 26, no. 2, pp. 209-236, 1978

[16] H. Masri, S. Krichen, and A. Guitouni, "A multi-start variable neighborhood search for solving the single path multicommodity flow problem," Applied Mathematics and Computation, vol. 251, pp. 132-142, 2015.

[17] L. R. Ford Jr and D. R. Fulkerson, "Constructing maximal dynamic flows from static flows," Operations research, vol. 6, no. 3, pp. 419433, 1958.

[18] L. Ford and D. R. Fulkerson, Flows in networks, vol. 1962. Princeton Princeton University Press, 1962.

[19] X. Liu, S. Mohanraj, M. Pióro, and D. Medhi, "Multipath routing from a traffic engineering perspective: How beneficial is it?", in Network Protocols (ICNP), 2014 IEEE 22nd International Conference on, pp. 143-154, IEEE, 2014 